\title{
Neurointensivmedizin: aneurysmatische Subarachnoidalblutung - State of the Art
}

Daniela Müller, Oliver Müller

\begin{abstract}
Die spontane, nicht traumatische Subarachnoidalblutung ist eine lebensbedrohliche Erkrankung, die mit vielfältigen Komplikationen und einer hohen Morbidität und Mortalität vergesellschaftet ist. In den ersten 24 Stunden hat die Ausschaltung der Blutungsursache Vorrang. Die Patienten sollen intensivmedizinisch überwacht und behandelt werden. Stringente Diagnostik und konsequente Behandlung verbessern das Outcome der Patienten nachweislich.
\end{abstract}

\section{Epidemiologie}

Die spontane Blutung in den intrakraniellen oder spinalen Liquorraum (nicht traumatische Subarachnoidalblutung, $\mathrm{SAB}$ ) ist für ca. $5 \%$ aller sog. Schlaganfälle verantwortlich. Die nicht traumatische Subarachnoidalblutung ist meist bedingt durch die Ruptur eines intrakraniellen Aneurysmas, selten einer arteriovenösen Malformation oder arteriovenösen Fistel. Dieser Artikel beschränkt sich auf die Behandlung der aneurysmatischen SAB. Die Inzidenz schwankt je nach Literaturangabe zwischen 2-22/ 100000 Einwohner. Das durchschnittliche Alter bei SAB liegt inzwischen bei 50-60 Jahren [1]. Statistisch sieht jeder Hausarzt 5-8 Patienten mit einer bislang nicht diagnostizierten SAB in seiner Praxis. Als gesicherte Risikofaktoren für die Entstehung zerebraler Aneurysmen und einer aneurysmatischen SAB gelten arterielle Hypertonie und Rauchen. Andere Risikofaktoren, die eine Arteriosklerose begünstigen, werden hinzugezählt. Eine familiäre Häufung ist beschrieben und begleitet die autosomaldominante polzystische Nierenerkrankung [2].

\section{Merke}

Eine nicht traumatische SAB stellt immer einen medizinischen Notfall dar: Die unverzögerte weiterführende Diagnostik und intensivmedizinische Therapie sowie die Behandlung der Ursache haben höchste Priorität.

Die prähospitale Mortalität der aneurysmatischen SAB Ausdruck der Dramatik der Erkrankung - ist mit 20\% unverändert hoch geblieben. Die 30-Tages-Mortalität beträgt etwa $35 \%$. Das funktionelle Überleben der Patienten hat sich aber in den vergangenen 30 Jahren durch ein besseres Verständnis der Pathophysiologie und moderne intensivmedizinische Behandlungskonzepte bedeutend verbessert: $70 \%$ besseres Outcome im Vergleich zu alten Fallserien [3].

\begin{tabular}{|ll}
\hline ABKÜRZUNGEN \\
ADH & antidiuretisches Hormon \\
CBF & zerebraler Blutfluss \\
CPP & zerebraler Perfusionsdruck \\
CCT & kraniale Computertomografie \\
CTA & computertomografische Angiografie \\
DCI & Delayed cerebral Ischemia \\
DI & Diabetes insipidus \\
DSA & digitale Subtraktionsangiografie \\
EVD & externe Ventrikeldrainage \\
GCS & Glasgow Coma Scale \\
HRST & Herzrhythmusstörungen \\
ICP & intrakranieller Druck \\
KAM & katheterassoziierte Meningitis \\
LP & Lumbalpunktion \\
MAD & mittlerer arterieller Druck \\
MRT & Magnetresonanztomografie \\
SAB & Subarachnoidalblutung \\
SIADH & Syndrom der inadäquaten ADH- \\
& Sekretion \\
ZNS & Zentralnervensystem \\
& \\
\hline
\end{tabular}

\section{Symptomatik}

Die Symptomatik der SAB ist typischerweise gekennzeichnet durch einen starken, in dieser Form noch nie aufgetretenen Kopfschmerz (Vernichtungskopfschmerz).

\section{Der Vernichtungskopfschmerz ist ein Hauptindikator der SAB und tritt in bis zu $80 \%$ der Fälle auf.}

Als weitere Symptome können Übelkeit und Erbrechen, Meningismus, vegetative Störungen und Krampfanfälle 
bis hin zur Bewusstlosigkeit auftreten. Wichtige Differenzialdiagnosen der SAB sind unter „Info - Differenzialdiagnosen der SAB“ genannt.

\section{INFO}

\section{Differenzialdiagnosen der SAB}

- Migräneattacke

- Glaukomanfall

- Dissektion der extrakraniellen A. vertebralis

- spinale SAB / spinales spontanes Hämatom

- ZNS-Tumorerkrankung

- Hydrozephalus anderer Genese (Foramen-Monroi-Blockade)

Vermutlich bis zu einem Viertel der Patienten erleidet im Vorfeld eine Warnblutung, die nicht richtig interpretiert oder aufgrund der geringer ausgeprägten Symptomatik bagatellisiert wird.

\section{Schweregrad}

Der Schweregrad der SAB wird üblicherweise nach der Graduierung nach Hunt und Hess und/ oder der Klassifikation der World Federation of Neurosurgical Societies (WFNS) eingeteilt ( $\triangleright$ Tab. 1). Ausmaß und Verteilung der Blutung werden in der kranialen Computertomografie (CCT) nach der Fisher-Skala oder deren Modifikation nach dem BNI-Score (Wilson; BNI: Barrow Neurological Institute) klassifiziert.

\section{Diagnostik}

Bei Verdacht auf eine SAB sollte als Standarddiagnostik immer eine native CCT durchgeführt werden [4]. Die Sensitivität der CCT in den ersten 24 Stunden ist hoch:
Sie beträgt ca. $93 \%$ und nach einer Woche immerhin noch $50 \%$. Im Rahmen dieser Untersuchung sollte unmittelbar eine computertomografische Angiografie (CTA) ergänzt werden zur Detektion einer Blutungsquelle. Die kraniale Magnetresonanztomografie (cMRT) ist aufgrund der zumeist schlechten oder instabilen klinischen Verfassung der Patienten und der langen Datenerfassungszeit in der Akutsituation nicht praktikabel. Zudem steht sie vielerorts nicht rund um die Uhr zur Verfügung ( Tab. 2) [5].

Sind die initiale CCT und/oder CMRT negativ, sollte bei hinreichendem Verdacht auf eine stattgehabte SAB eine Liquorpunktion (LP) durchgeführt werden. Diese sollte standardisiert unter sterilen Kautelen erfolgen. Um eine artifizielle Blutbeimengung auszuschließen, wird das Vorhandensein von Blut im Liquor über eine 3-Gläser-Probe bestimmt. Zusätzlich können Siderophagen (Makrophagen, die Hämosiderin phagozytiert haben) in der zytologischen Untersuchung des Liquors gefunden werden, sollte die Blutung älter als 36 Stunden sein. Diese können noch Wochen nach einer Blutung nachweisbar sein.

\section{Merke}

Die digitale Subtraktionsangiografie der intrakraniellen und extrakraniellen Blutgefäße (DSA) ist nach wie vor Goldstandard in der Diagnostik der Subarachnoidalblutung [6].

Bei nachgewiesener SAB und initial negativer DSA sollte sie nach 10-14 Tagen wiederholt werden. Gleichzeitig lässt sich durch die DSA auch interdisziplinär der beste Weg einer Aneurysmaversorgung für den Patienten festlegen [7] ( $\triangleright$ Abb. 1). Trotz dieser ausführlichen Diagnostik kann jedoch in ca. $10 \%$ der Fälle keine Blutungsursache gefunden werden.

-Tab. 1 Einteilung der Subarachnoidalblutung.

\begin{tabular}{|c|c|c|c|c|}
\hline GCS & WFNS & $\begin{array}{l}\text { neurologi- } \\
\text { sches Defizit }\end{array}$ & Hunt und Hess & klinische Zeichen der Hunt-und-Hess-Einteilung \\
\hline 15 & 1 & $\mathrm{~N}$ & 1 & $\begin{array}{l}\text { diskreter Kopfschmerz / Meningismus, } \\
\text { asymptomatisch }\end{array}$ \\
\hline $14 / 13$ & II & $\mathrm{N}$ & II & $\begin{array}{l}\text { starker Kopfschmerz / Meningismus, ggf. Hirnner- } \\
\text { venauffälligkeiten, keine andere Fokalneurologie }\end{array}$ \\
\hline $14 / 13$ & III & J & III & $\begin{array}{l}\text { Somnolenz, Desorientiertheit, milde } \\
\text { Fokalneurologie }\end{array}$ \\
\hline $12-7$ & IV & NA & IV & $\begin{array}{l}\text { Stupor, deutliche bis schwere Hemiparese, vegeta- } \\
\text { tive Entgleisung, beginnende } \\
\text { Einklemmungserscheinung }\end{array}$ \\
\hline $6-3$ & V & NA & V & $\begin{array}{l}\text { tiefes Koma, deutliche Zeichen der Einklemmung, } \\
\text { moribunde Erscheinung }\end{array}$ \\
\hline
\end{tabular}


$>$ Tab. 2 Diagnostik der Subarachnoidalblutung.

\begin{tabular}{|l|l|l|}
\hline Untersuchung & Sensitivität / Spezifität & Vorteile / Nachteile \\
\hline CCT & hohe Sensitivität & $\begin{array}{l}\text { Vorteile: große Verfügbarkeit, Ergänzung einer CT-Angio- } \\
\text { grafie möglich }\end{array}$ \\
\hline MRT & hohe Sensitivität, hohe Spezifität & $\begin{array}{l}\text { Nachteile: lange Untersuchungsdauer, Untersuchung beat- } \\
\text { meter Patienten schwierig }\end{array}$ \\
\hline LP & hohe Sensitivität & Nachteil: falsch positive Befunde (3-Gläser-Probe!) \\
\hline DSA & höchste Sensitivität, höchste Spezifität & Vorteil: Behandlung der Blutungsquelle in einem \\
\hline CCT: kraniale Computertomografie, DSA: digitale Subtraktionsangiografie, LP: Lumbalpunktion,MRT: Magnetresonanztomografie
\end{tabular}

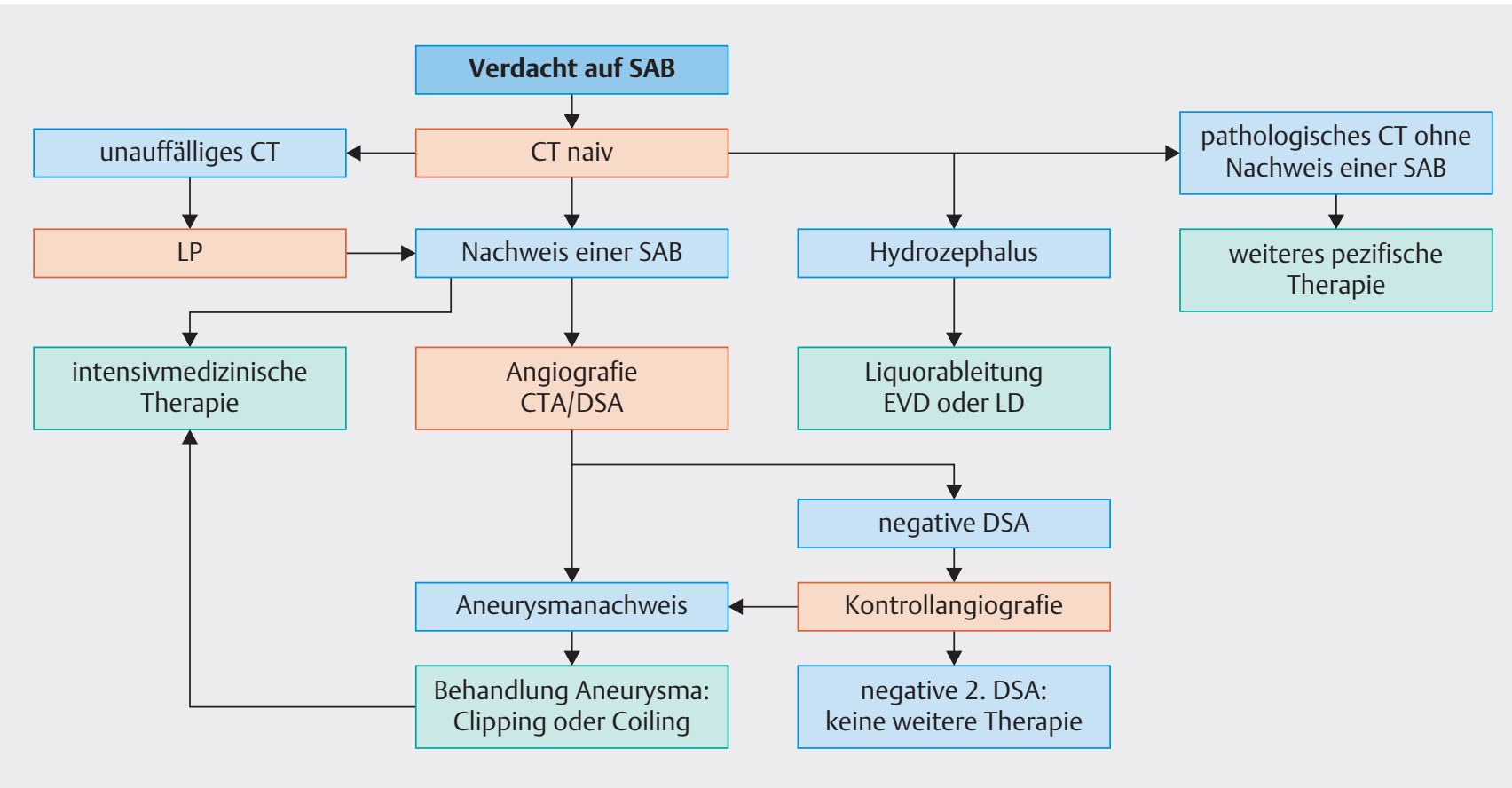

Abb. 1 Diagnostisches und therapeutisches Vorgehen bei aneurysmatischer Subarachnoidalblutung. CT: Computertomografie, CTA: computertomografische Angiografie, DSA: digitale Subtraktionsangiografie, EVD: externe Ventrikeldrainage, LD: Lumbaldrainage, LP: Lumbalpunktion, SAB: Subarachnoidalblutung.

\section{Therapie und Komplikationen}

\section{Präinterventionelle Therapie}

Jede neu diagnostizierte $S A B$ sollte zunächst auf einer Intensivstation mit neurologisch/neurochirurgischer Expertise behandelt werden - zumindest bis eine Blutungsquelle ausgeschlossen wurde. Ist der Patient wach, sind Katheteranlagen vor Versorgung einer Blutungsquelle zu vermeiden, um Stressphasen zu minimieren. Zielwert für den systolischen Blutdruck sind moderate $160 \mathrm{mmHg}$. Gleichzeitig muss der zerebrale Perfusionsdruck aufrechterhalten werden, sodass der mittlere arterielle Druck (MAD) $60-70 \mathrm{mmHg}$ nicht unterschreiten sollte. Eine hinreichende Analgesie ist wichtig. Vorrangig sind potente Analgetika wie beispielsweise Metamizol oder Morphine (z. B. MSI s. c. oder i. v.) zu wählen, die parenteral verabreicht werden. Vor Diagnostik und eventueller Versorgung der Blutungsquelle muss der Patient nüchtern bleiben. Auf ausreichende Flüssigkeitszufuhr ist zu achten [2].

Merke

Es ist auf eine hinreichende zerebrale Perfusion und eine ausreichende Analgesie vor Versorgung der Blutungsquelle zu achten.

\section{Initiale Begleitkomplikationen}

\section{Hydrozephalus}

Die häufigste Komplikation der SAB stellt mit bis zu 30\% der Hydrozephalus dar. Er ist durch eine Blutung in die inneren (Hydrocephalus occlusus) oder äußeren Liquorräume (Hydrocephalus malresorptivus) bedingt. Die Behandlung des Hydrozephalus hat Vorrang vor u. U. langwieriger Diagnostik (s. „Fallbeispiel - Symptome und 
Diagnostik“). Die Anlage einer externen Ventrikeldrainage (EVD) beherrscht nicht nur diese Komplikation, sondern erlaubt gleichzeitig ein Monitoring des Hirndrucks und damit des zerebralen Perfusionsdrucks (CPP). Die operative Anlage der EVD sollte stets in Intubationsnarkose erfolgen, um die Stresssituation für den Patienten zu minimieren. In ausgewählten Fällen kann statt der intraventrikulären Liquorableitung eine Lumbaldrainage gelegt werden. Hier ist sorgfältig abzuwägen, ob ein Hydrocephalus occlusus vorliegt: In diesem Fall kann die Anlage einer Lumbaldrainage zur unteren Einklemmung durch Herniation des Kleinhirns in das Foramen magnum führen. Bei Anlage der Lumbaldrainage ist auf eine hinreichende Analgesie und ggf. Sedierung des Patienten zu achten.

\section{Kardiale Ereignisse}

Zweithäufigste Komplikation (ca. 14\%) der SAB sind kardiale Ereignisse, die sich als Myokardinfarkt oder Herzrhythmusstörungen (HRST) manifestieren können [8] (s. „Fallbeispiel - Symptome und Diagnostik“). Ihr Auftreten korreliert mit dem Schweregrad der SAB und verschlechtert das Outcome der Patienten hinsichtlich Morbidität und Mortalität dramatisch. Im Rahmen einer SAB kommt es zu einer sympathischen Aktivierung mit exzessiver Katecholaminausschüttung. Besonders in den ersten 48 Stunden nach Blutungsereignis treten HRST und Troponin- I-Erhöhungen auf.

\section{Cave}

Herzrhythmusstörungen und erhöhte Troponin-IWerte führen nicht selten dazu, dass SAB-Patienten zunächst einer kardialen Diagnostik zugeführt werden.

\section{FALLBEISPIEL}

\section{Symptome und Diagnostik}

Ein 34-jähriger Patient stellt sich in der Notaufnahme vor nach postkoital einsetzenden stärksten Kopfschmerzen. Vorerkrankungen sind keine bekannt, die neurologische Untersuchung ist zunächst unauffällig. Bei initialen Herzrhythmusstörungen im AufnahmeEKG wird zunächst eine kardiale Ursache vermutet. Das Troponin I ist aber im Aufnahmelabor unauffällig und im weiteren Verlauf erscheint der Patient zunehmend bewusstseinsgetrübt, sodass ein natives Schädel- CT durchgeführt wird. In den Aufnahmen sieht man eine basal betonte $S A B$ (roter Pfeil in $>$ Abb. 2), verdächtig auf eine aneurysmatische Blutung. Es finden sich betonte Temporalhörner als Zeichen eines beginnenden Hydrozephalus (grüner Pfeil in -Abb. 2). Bei nun inzwischen somnolentem Patienten wird vor weiterer Diagnostik eine EVD zur Liquorableitung und Hirndruckmessung angelegt.

Die Bandbreite kardialer Symptome erstreckt sich von einem erhöhten Troponin-I-Spiegel über EKG-

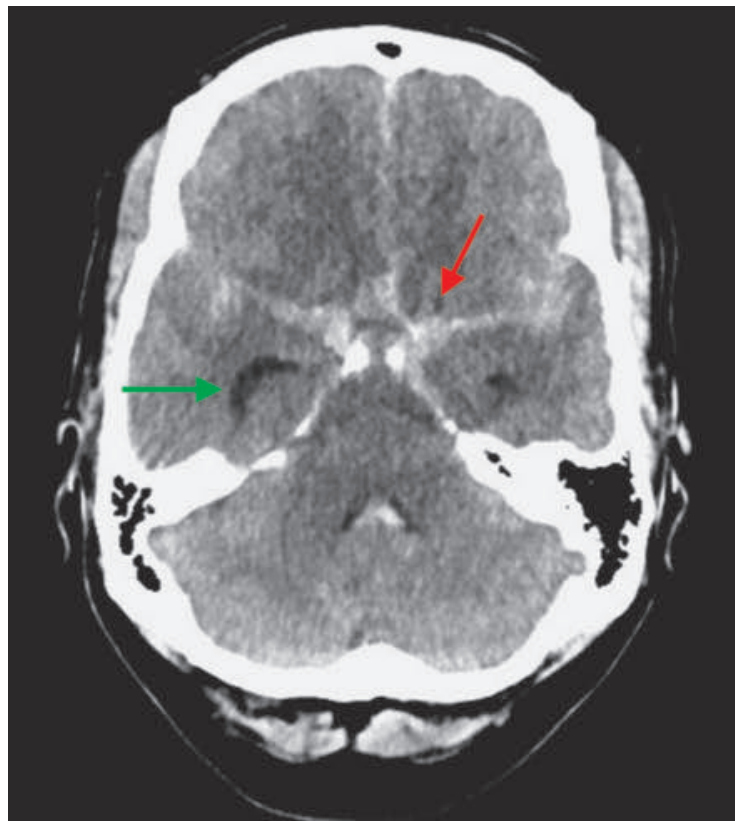

Abb. 2 Die kraniale Computertomografie zeigt eine basal betonte Subarachnoidalblutung (roter Pfeil) und als Zeichen eines beginnenden Hydrozephalus betonte Temporalhörner (grüner Pfeil).

Veränderungen und Rhythmusstörungen bis hin zu hämodynamisch relevanten Wandbewegungsstörungen in der Echokardiografie. Die sog. neurogene Stresskardiomyopathie (Tako-Tsubo-Kardiomyopathie) kann in eine kardiale Dekompensation münden [8].

\section{Weitere Komplikationen}

Die SAB verursacht bei einem Teil der Patienten zerebrale Krampfanfälle. Eine Empfehlung zur prophylaktischen antikonvulsiven Therapie gibt es auf Basis der Literatur nicht. Man muss jedoch bei Patienten mit einem persistierend schlechten neurologischen Status in Erwägung ziehen, dass ein nonkonvulsiver Status epilepticus vorliegen kann. Hier ist eine Elektroenzephalografie zum Ausschluss zu empfehlen.

Die konsequente Behandlung einer vorbestehenden Hyperglykämie und/oder Hyperthermie verringert Sekundärkomplikationen (Folgeinfektionen) und kann das Outcome der Patienten verbessern [2].

Eine Komplikation, die initial zumeist unerkannt verläuft, ist das Terson-Syndrom. Die Einblutung in den Glaskörper beeinträchtigt die Patienten im Verlauf der Erkrankung durch Visusminderung und kann zum Sekundärglaukom oder zur Amblyopie führen. Die Spontanremission ist häufig, jedoch ist u. U. eine Vitrektomie erforderlich. Daher wird eine augenärztliche Untersuchung vor Entlassung des Patienten in die Rehabilitation oder häusliche Umgebung empfohlen [9] ( Tab. 3). 
- Tab. 3 Komplikationen der Subarachnoidalblutung.

\begin{tabular}{|l|l|l|}
\hline Komplikationen & Auftreten & Tag \\
\hline Hydrocephalus occlusus & $\begin{array}{l}\text { mit Blutungsereignis durch Verlegung der } \\
\text { Liquorablaufwege }\end{array}$ & 0,5 \\
\hline Hydrocephalusmalresorptivus & $\begin{array}{l}\text { im Verlauf durch gestörte Liquorresorption nach } \\
\text { Abbau der Blutung }\end{array}$ & $10-30$ \\
\hline kardiale Ereignisse & $\begin{array}{l}\text { im Zusammenhang mit Ereignis oder imkurzfristi- } \\
\text { gen Verlauf durch erhöhten ICP }\end{array}$ & $1-3$ \\
\hline neurogenes Lungenödem & $\begin{array}{l}\text { nach Blutung durch ICP-Anstieg und } \\
\text { Sympathikusaktivierung }\end{array}$ & $2-5$ \\
\hline andere pulmonale Ereignisse & $\begin{array}{l}\text { Aspirations- / Beatmungspneumonien, Atelektasen, } \\
\text { Pleuraergüsse }\end{array}$ & $3-14$ \\
\hline DCI & transiente Ischämien durch gestörten CBF und CPP & $(2) 4-14(21)$ \\
\hline Terson-Syndrom & $\begin{array}{l}\text { mit initialem Ereignis durch erhöhten ICP, zumeist } \\
\text { erst spät diagnostiziert }\end{array}$ & 1 \\
\hline Elektrolytstörungen & SIADH, CSW, DI & $4-21$ \\
\hline \begin{tabular}{l} 
CBF: Cerebral Blood Flow / zerebraler Blutfluss, CPP: Cerebral Perfusion Pressure / zerebraler Perfusionsdruck, CSW: Cerebral Salt Wasting \\
Syndrome / zerebrales Salzverlustsyndrom, DCI: Delayed cerebral Ischemia / verzögert auftretendes neurologisches Defizit, DI: Diabetes insi- \\
\hline pidus, ICP: Intracranial Pressure / intrakranieller Druck, SIADH: Syndromder inadäquaten ADH-Sekretion, ADH: antidiuretisches Hormon
\end{tabular}
\end{tabular}

\section{Behandlung des Aneurysmas}

Aneurysmen der zerebralen Arterien liegen zu 85\% im vorderen Stromkreis. Im Rahmen der Diagnostik werden bei $15 \%$ der Patienten multiple Aneurysmen gefunden. Während für asymptomatische Aneurysmen die Leitlinien eine Behandlungsempfehlung ab $7 \mathrm{~mm}$ maximalem Durchmesser aussprechen, müssen rupturierte Aneurysmen ungeachtet der Größe immer behandelt werden. Der Behandlungsalgorithmus für Aneurysmen sollte dabei in jeder Klinik interdisziplinär in einem neurovaskulären Team abgestimmt werden. Dieses Team hält beide Therapieoptionen vor - die endovaskuläre Versorgung und das mikrochirurgische Clipping - und verfügt über die erforderliche neurointensivmedizinische Kompetenz. Die Entscheidung über die beste Aneurysmaversorgung - neurochirurgisches Clipping oder endovaskuläre Therapie - sollte nach Durchführung einer DSA interdisziplinär getroffen werden [5] (s. „Fallbeispiel - Therapie des Aneurysmas").

Die Nachblutung ist die am meisten gefürchtete Komplikation der SAB, die mit hoher Morbidität und Mortalität einhergeht. Risikofaktoren für eine Nachblutung sind

- höheres Lebensalter,

- hoher Hunt-und-Hess-Grad,

- eine Aneurysmagröße > 10 mm sowie

- ein systolischer Blutdruck > $160 \mathrm{mmHg}$.

\section{Merke}

Aufgrund des Risikos einer frühen Nachblutung, die die schwerwiegendste SAB-Komplikation darstellt, sollte eine frühzeitige Aneurysmaversorgung innerhalb der ersten 48 Stunden angestrebt werden [10].
Unklar ist nach aktueller Datenlage, ob eine Aneurysmaversorgung in der sog. Vasospasmusphase, die etwa ab Tag 5 postiktal beginnt, einen negativen Einfluss auf das Outcome hat [11].

\section{Behandlung nach Aneurysmaversorgung}

Die Therapie der Patienten mit einer SAB erfolgt auch nach Ausschaltung des Aneurysmas weiter auf einer Intensivstation, die über diese organspezifische, also Neurointensiv- Kompetenz verfügt [12].

Die Therapie der SAB zielt hierbei in erster Linie auf die Überwachung des Patienten ab, um mögliche Komplikationen frühzeitig zu erkennen und gezielt zu behandeln. Zur intensivmedizinischen Überwachung gehört die 2-stündliche Beurteilung des Neurostatus des Patienten anhand

- des Glasgow Coma Score (GCS),

- der World Federation of Neurological Surgeons (WFNS) Scale sowie

- der Richmond Agitation Sedation Scale (RASS).

\section{FALLBEISPIEL}

\section{Therapie des Aneurysmas}

In der anschließend durchgeführten DSA zeigt sich bei unserem Patienten als Ursache der Blutung ein Aneurysma der A. communicans anterior (roter Pfeil in - Abb. 3a).Weitere Aneurysmen finden sich nicht. Das Aneurysma weist eine breite Basis des Sacks auf, was ein Coiling schwierig und ein zusätzliches Stenting wahrscheinlich macht (grüner Pfeil in \ Abb. 3b). Interdisziplinär wird daher zwischen Neurochirurgen und Neuroradiologen entschieden, das Aneurysma noch 
am Aufnahmetag chirurgisch mittels Clipping zu versorgen. Aufgrund des über die EVD gemessenen erhöhten intrakraniellen Druckes wird entschieden, gleichzeitig eine dekompressive Kraniektomie durchzuführen.Der Patient wird nach der Angiografie direkt in den OP gebracht. Dort wird das Aneurysma komplikationslos mittels Clip ausgeschaltet.

Der Status der Pupillomotorik wird ebenfalls - je nach intrakraniellen Druckverhältnissen - mindestens 2stündlich erfasst. Die kontinuierliche Messung des intrakraniellen Drucks (ICP) ist bei Patienten mit einem pathologischen Befund in der kranialen Bildgebung erforderlich, wenn dieser Befund eine abnorme Druckerhöhung wahrscheinlich macht. Die ICP-Messung über eine EVD erlaubt gleichzeitig eine Liquorabnahme zur Senkung des Hirndrucks nach der Monro-Kellie-Doktrin und eine Analyse des Liquors zum Infektionsnachweis. Alternativ kann der ICP über intrazerebrale Sonden bestimmt werden, die im Parenchym und nicht im Ventrikelsystem liegen. Sie ermöglichen aber keine Liquorentnahme. Der Stellenwert der kontinuierlichen Mikrodialyse ist umstritten. Der Aufwand der Dialysatbestimmung korreliert nicht mit dem Nutzen für eine zeitnahe Therapieentscheidung.

Zahlreiche Studien haben in der letzten Dekade die Behandlung der SAB vor und nach Aneurysmaversorgung nachhaltig verändert. Dadurch wurde das Outcome der Patienten um $70 \%$ verbessert. So wurde die prophylaktische Behandlung mit Hypervolämie, gezielter Hypertension und Hämodilution (Triple-H-Therapie) zugunsten eines individuellen, sequenziellen und bedarfsangepassten Maßnahmenbündels aufgegeben [13].

\section{Merke}

Die umgehende Verlegung und Überwachung der Patienten auf einer Intensivstation erlaubt das zeitnahe Gegensteuern, wenn Komplikationen wie HRST, ICPErhöhung oder DCI drohen, die Patienten zu gefährden.

\section{Zerebrale Durchblutung und intrakranieller Druck in der Frühphase}

Das in den Subarachnoidalraum austretende Blut verursacht eine Massenwirkung intrakraniell, der zufolge - nach der Monro-Kellie-Doktrin - die beiden anderen Kompartimente (Gehirn und Liquor) abnehmen müssen. Es gibt zwar keine gesicherten Daten über den intrakraniellen Druckverlauf während der initialen Ruptur eines Aneurysmas bei Patienten. Die Druckspitzen des ICP werden jedoch regelmäßig beobachtet, wenn es vor Versorgung des Aneurysmas zu einer Nachblutung kommt. Der zerebrale Blutfluss (CBF) nimmt in dieser Situation dramatisch ab (um bis zu $80 \%$ ) und erholt sich erst allmählich, wenn die Autoregulation der zerebralen Perfusion greift. Die

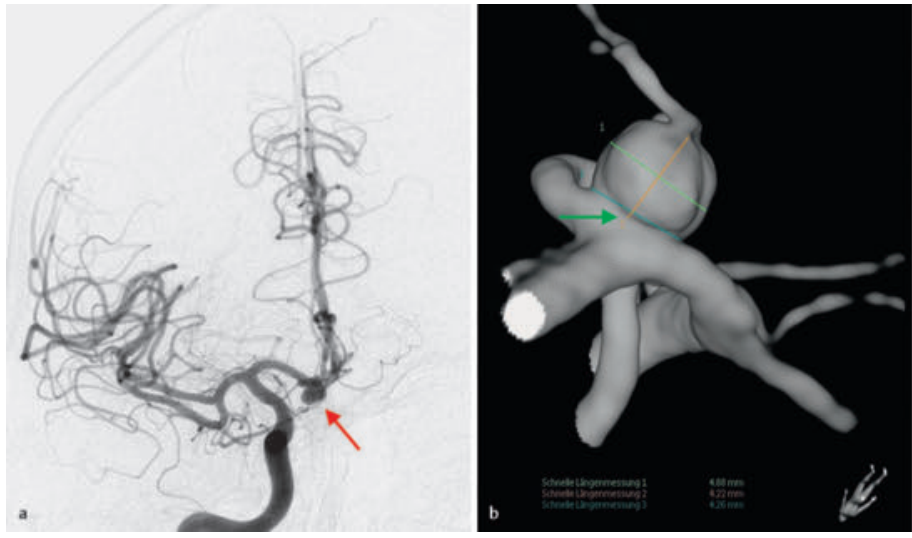

- Abb. 3 Digitale Subtraktionsangiografie nach Subarachnoidalblutung. a Als Ursache der Blutung findet sich ein Aneurysma der A. communicans anterior (roter Pfeil). b Die breite Basis des Aneurysmas (grüner Pfeil) macht ein Coiling schwierig, sodass die Entscheidung für ein chirurgisches Clipping getroffen wird.

Folge ist eine globale Minderperfusion des Gehirns, die eine Minderoxygenierung des Gehirnparenchyms nach sich zieht. Besonders berücksichtigt werden muss, dass die Patienten aus völliger Ahnungslosigkeit eine SAB erleiden, eine adäquate Sauerstoffpartialsättigung des Gewebes also niemals vorausgesetzt werden kann. Die Sauerstoffunterversorgung führt ihrerseits wieder zu einer Ausschüttung von Akute-Phase-Proteinen und einer konsekutiven Hirnschwellung, die den CBF weiter kompromittiert. Während sich die Perfusion nach zerebraler Ischämie schnell wieder restituieren kann, ist dies nach einer SAB unter Umständen langwierig und abhängig vom erhöhten ICP [14].

Durch die Ausschaltung des Aneurysmas wurde die Nachblutungsgefahr minimiert. Das Blutungsereignis hat jedoch eine Kaskade in Bewegung gesetzt (s. „Info Early Brain Injury“), deren Auswirkung den Patienten in den nächsten Wochen unmittelbar bedroht und langfristige Veränderungen nach sich ziehen kann.

\section{INFO}

\section{Early Brain Injury}

In der unmittelbaren Frühphase nach Blutung kommt es zu einer Konstriktion der kleinsten zerebralen Gefäße, Formation von Mikrothromben und einer partiellen Auflockerung der Blut-Hirn-Schranke. Diese werden durch inflammatorische Prozesse initiiert und unterhalten. Pathophysiologisch kennzeichnet dieser Impact eine Early Brain Injury in der Frühphase der SAB. Die Entzündungskaskade führt zusammen mit der fokalen Ischämie und dem Ödem zu einer IonenDysbalance, die eine synchrone Entladung der Neurone fördert. Diese breitet sich als „spreding depolarization“ über den Kortex aus und unterhält ihrerseits die fokale Ischämie und das Ödem [1]. 


\section{Delayed cerebral Ischemia und / oder angiografischer Vasospasmus}

Verengungen der größeren Hirnarterien (Vasospasmus) treten nach SAB ab dem 2. Tag auf, das Maximum wird zwischen Tag 6 und 9 erreicht. Die Gefäßweite normalisiert sich zumeist wieder ab Tag 14. Angiografisch werden bei bis zu $70 \%$ der Patienten Vasospasmen nachgewiesen. Dieses Phänomen wurde von Eckert und Riemenschneider bereits 1951 beschrieben. Lange Zeit wurde der angiografische Vasospasmus mit einer neurologischen Verschlechterung der Patienten gleichgesetzt. Inzwischen ist durch Studien belegt, dass Spasmen der Hirngefäße erst klinisch relevant werden, wenn es zu einer Reduzierung des Gefäßvolumens > 70 \% kommt. Gleichzeitig treten neurologische Verschlechterungen bei Patienten auch ohne Nachweis eines zerebralen Vasospasmus auf. Vasospasmus und DCl sind keine Synonyme und sollten auch nicht als solche verwendet werden, da die $\mathrm{DCl}$ eine klinische Diagnose ist, der Vasospasmus eine rein radiologische.

Die klinisch manifeste verzögerte zerebrale Ischämie (DCI) infolge eines höhergradigen Vasospasmus betrifft bis zu 30 \% der Patienten im Verlauf der Hospitalisierung. 2010 wurde von einem Research Committee ein Vorschlag für eine Definition der DCl in Stroke publiziert [15] (s. „Definition - Delayed cerebral Ischemia“). Der Hintergrund war, auch in Studien eine einheitliche Beurteilung des Outcome von Patienten mit SAB zu gewährleisten.

\section{DEFINITION}

Delayed cerebral Ischemia

- eine neurologische Verschlechterung des Patienten (fokales neurologisches Defizit, Hemiparese, Aphasie, Apraxie oder Ähnliches) oder

- eine Abnahme des GCS um > 2 Punkte (entweder global oder in einer einzelnen Kategorie)

Diese Untersuchungsbefunde sollen über eine Stunde nachvollziehbar und nicht durch andere klinische oder radiologische (CT oder MRT) Befunde erklärbar sein. Sie dürfen auch nicht im unmittelbaren Nachgang der Versorgung des Aneurysmas aufgetreten sein [15].

\section{Merke}

Die DCI tritt bei bis zu $30 \%$ der Patienten nach SAB auf. Sie durch kontinuierliche Überwachung der Patienten rechtzeitig zu erkennen, ermöglicht die Initiierung spezifischer Therapien.

\section{Detektion des zerebralen Vasospasmus}

Zur Detektion eines zerebralen Vasospasmus möglichst vor einer DCl sollte täglich eine Doppler-Sonografie der Hirngefäße durchgeführt werden. Die Doppler-
Untersuchung ist zwar wenig sensitiv und spezifisch, jedoch eine nicht invasive, schnelle und kosteneffektive Untersuchung. Als dringend verdächtig auf eine signifikante Einengung des Gefäßlumens gilt

- eine Erhöhung der Fließgeschwindigkeit in den zerebralen Arterien von $200 \mathrm{~cm} / \mathrm{s}$ und mehr oder

- eine Erhöhung derselben um $100 \%$ innerhalb von $24 \mathrm{~h}$.

Diese Befunde sollten weiter angiografisch abgeklärt werden.

Perfusionsuntersuchungen zur Beurteilung des CBF sind ebenfalls sensitive Untersuchungen. Der logistische Aufwand ist jedoch bedeutend größer als bei der repetitiven Doppler-Untersuchung. Zudem sind Perfusionsuntersuchungen zumeist - nicht zuletzt aufgrund der Strahlenbelastung - nicht sinnvoll seriell als Screening-Untersuchung einsetzbar. Sie sollten daher nur bei hinreichendem klinischem Verdacht durchgeführt werden.

\section{Prophylaxe der DCl}

Zur Primärprophylaxe der DCl steht als einziges Medikament mit nachgewiesener Wirksamkeit Nimodipin zur Verfügung, das in der Dosierung $6 \times 60 \mathrm{mg}$ oral gegeben wird [2]. Aktuell wird in einer placebokontrollierten, verblindeten, randomisierten Multicenterstudie die intrathekale Wirksamkeit von Nimodipin gegen die orale Form getestet (Newton-II-Studie).

Zahlreiche andere Medikamente (Magnesium, Statine u. a.) konnten in mehr als 30 klinischen Studien keinen Wirksamkeitsnachweis zur Verhinderung der $\mathrm{DCl}$ erbringen. Für den Endothelin-Antagonisten Clazosentan laufen noch Studien, sodass für diese Substanz noch keine abschließende Beurteilung vorliegt.

Möglicherweise wurde der Fokus in der DCl-Therapie zu sehr auf den arteriellen Schenkel der zerebralen Gefäße gelegt. Es gibt Überlegungen, dass das Pooling in den venösen Hirngefäßen und eine Abnahme der Flussgeschwindigkeit in den arteriellen Gefäßen eine weitere Ursache der DCl sein könnten. 2013 führte die Gruppe von J. Marc Simard einen Therapieversuch mit kontinuierlicher Low-Dose-Heparin-Infusion in einer Proof-of-Concept- Untersuchung durch: Es zeigte sich ein besseres Outcome der Patienten in Bezug auf DCl in der Heparin-Gruppe [16]. Andere Arbeitsgruppen konnten diese Ergebnisse allerdings nicht in der Form reproduzieren.

\section{Merke}

Aufgrund der aktuellen Studienlage gilt orales Nimodopin $(6 \times 60 \mathrm{mg})$ als die einzige wirksame Prophylaxe der DCl. 
Therapie der DCI

Kommt es zu einer DCl, verbunden mit einer Hypoperfusion im abhängigen Stromgebiet, so wird zunächst eine induzierte Hypertonie eingeleitet. Der angestrebte Zielwert des MAD sollte bei $>90 \mathrm{mmHg}$ liegen. Studien haben gezeigt, dass die induzierte arterielle Hypertension ausschlaggebend für die Verbesserung der zerebralen Perfusion ist. Sie ist als einziger Bestandteil der früher regelmäßig eingesetzten Triple$\mathrm{H}$-Therapie übriggeblieben. Die induzierte Hypertonie soll - wenn erforderlich - durch den Einsatz von Katecholaminen, hier bevorzugt durch Noradrenalin, erreicht werden.

Die prophylaktische Durchführung der induzierten Hypertonie, Hypervolämie und Hämodilution hat sich als nicht sinnvoll erwiesen [13].

\section{INFO \\ Endovaskuläre Therapien}

Bei angiografisch nachgewiesenem hämodynamisch wirksamem Vasospasmus können auch endovaskuläre Verfahren zum Einsatz kommen. Hier steht die intraarterielle Gabe von vasodilatatorischen Medikamenten - Nimodipin oder Papaverin - an erster Stelle. Sie wird teils als Bolus, bisweilen auch als kontinuierliche Infusion über liegende Katheter durchgeführt. Höhergradige kurzstreckige Stenosen können auch mit einer Ballondilatation wiedereröffnet werden. Die Gefahr einer Gefäßwandruptur ist gegeben. Diese Behandlungen entstammen keiner Leitlinie und bedürfen aufgrund der ungenügenden Evidenz für die Wirksamkeit einer interdisziplinären Einzelfallentscheidung.

\section{Elektrolytstörungen}

Elektrolytstörungen nach SAB sind eine häufige Komplikation, vor allem betreffen diese den Natriumhaushalt. Die Hyponatriämie $\left(\mathrm{Na}^{2+}<130 \mathrm{mmol} / \mathrm{l}\right)$ steht dabei im Vordergrund. Zurückzuführen ist sie auf

- ein zerebrales Salzverlustsyndrom (cerebral salt wasting syndrome: CSW) oder

- eine inadäquate ADH-Sekretion (Syndrom der inadäquaten ADH-Sekretion: SIADH).Möglich ist auch eine Kombination beider Ursachen bei einem Patienten. Diese Störungen des Elektrolythaushalts treten vor allem bei der SAB aus Aneurysmen des vorderen Kreislaufes auf. Sie manifestieren sich häufig am Ende der 1. Woche nach SAB.

Die Hyponatriämie sollte mit hypertoner $\mathrm{NaCl}$-Lösung ausgeglichen werden. Eine Flüssigkeitsrestriktion - sonst eine adäquate Therapie bei Hyponatriämie durch SIADH - ist hier keine Therapiealternative aufgrund der erhöhten Gefahr einer transienten Hypotension oder von Mikrothrombenbildung. Fludrocortison kann die Natriurese reduzieren und kann zur Begrenzung des CSW eingesetzt werden. Vaptane als neue Medikamente zur Behandlung der Hyponatriämie sind bis jetzt nur oral verfügbar und die Wirkung ist bei der SAB noch nicht ausreichend belegt.

Die Hypernatriämie $\left(\mathrm{Na}^{2+}>145 \mathrm{mmol} / \mathrm{l}\right)$ ist oftmals iatrogen bedingt als Folge von Infusionstherapien mit Mannitol oder hypertonen Kochsalzlösungen zur ICPKontrolle. Seltener tritt infolge der SAB ein Diabetes insipidus (DI) auf. Zumeist liegt ein zentraler DI vor. Die Korrektur des Serumnatrium-Wertes erfolgt durch Infusion $5 \%$ iger Glukoselösungen oder halbisotonischer $\mathrm{NaCl}$-Lösungen. Die Normalisierung des Wertes sollte dabei $0,5 \mathrm{mmol} / \mathrm{l}$ pro Stunde nicht überschreiten. Der zentrale DI spricht auch auf die Gabe von synthetischem ADH an.

\section{Merke}

Die Elektrolytstörungen sind eine ernst zu nehmende Komplikation und bedürfen einer engmaschigen Überwachung der Patienten sowie der Laborwerte und einer überlegten Gegensteuerung.

\section{Fieber}

Fieber ist bei der SAB mit schlechterem Outcome assoziiert. Bei Fieber aufgrund eines infektiösen Geschehens muss die Infektion frühzeitig und konsequent behandelt werden. Bei der Fokussuche darf eine katheterassoziierte Meningitis (KAM) bei liegender EVD oder Parenchymsonde nicht übersehen werden. Die KAM tritt bei SAB deutlich häufiger auf und erhöht die Rate dauerhafter Liquorresorptionsstörungen.

Durch die SAB kann ein zentrales Fieber infolge der Affektion des Hypothalamus entstehen. Dies muss gegen ein infektiöses Geschehen abgegrenzt werden. Therapeutisch sollte eine Normothermie auch bei zentralem Fieber angestrebt werden. Hierzu können gängige Antipyretika und physikalische Maßnahmen eingesetzt werden. Bei Gabe von Metamizol und Paracetamol i. v. ist auf eine ausreichende Einhaltung des MAD zu achten.

\section{Begleitende Anämie}

Während der Intensivbehandlung sollte bei den Patienten ein Hb-Wert von $8-10 \mathrm{mg} / \mathrm{dl}$ angestrebt werden. Dies bedeutet, dass ein niederschwelligerer Transfusionstrigger gilt als die bei anderen Patientengruppen zugrunde gelegten $7 \mathrm{mg} / \mathrm{dl}$. Die Aufklärung über die Gabe von Blutprodukten oder 
Blutbestandteilen erfolgt unbenommen nach den Vorgaben des Transfusionsgesetzes.

\section{Persistierender Hydrozephalus und Shuntpflichtigkeit}

Patienten, die initial nach SAB eine externe Liquorableitung benötigen, sind zu 20-25\% auf eine dauerhafte Liquorableitung angewiesen. Sie entwickeln als Folge der SAB einen Hydrocephalus malresorptivus durch eine inflammatorische Obliteration der Resorptionswege des Liquors u. a. an den Pacchioni-Granulationen [17]. Die EARLYDRAIN-Studie hat untersucht, ob eine frühe lumbale Drainage die Rate dauerhafter Shuntpflichtigkeit nach SAB reduzieren kann. Die Ergebnisse werden für dieses Jahr erwartet [18]. Der persistierende Hydrocephalus malresorptivus wird durch Implantation eines ventrikuloperitonealen (VP) Shunts behandelt (s. „Fallbeispiel - Postoperativer Verlauf“). Andere Ableitungsorte (kardial, pleural etc.) sind nicht die erste Wahl.

\section{Merke \\ Das frühzeitige Erkennen der Shuntpflichtigkeit kann bei den betroffenen Patienten das Auftreten einer katheterassoziierten Meningitis verringern [19]. \\ Pulmonale Komplikationen und neurogenes Lungenödem}

Affektionen der Lunge treten in retrospektiven Studien bei mehr als $30 \%$ der Patienten mit SAB auf. Hierunter sind respiratorassoziierte Pneumonien, Pleuraergüsse und Atelektasen genauso subsumiert wie das neurogene Lungenödem. Letzteres tritt vermutlich in einer Häufigkeit von 2-8\% auf [20], es liegen jedoch keine prospektiven Untersuchungen hierzu vor. Die Ursache des neurogenen Lungenödems ist unklar. Es wird eine Folge der überschießenden Sympathikusaktivierung
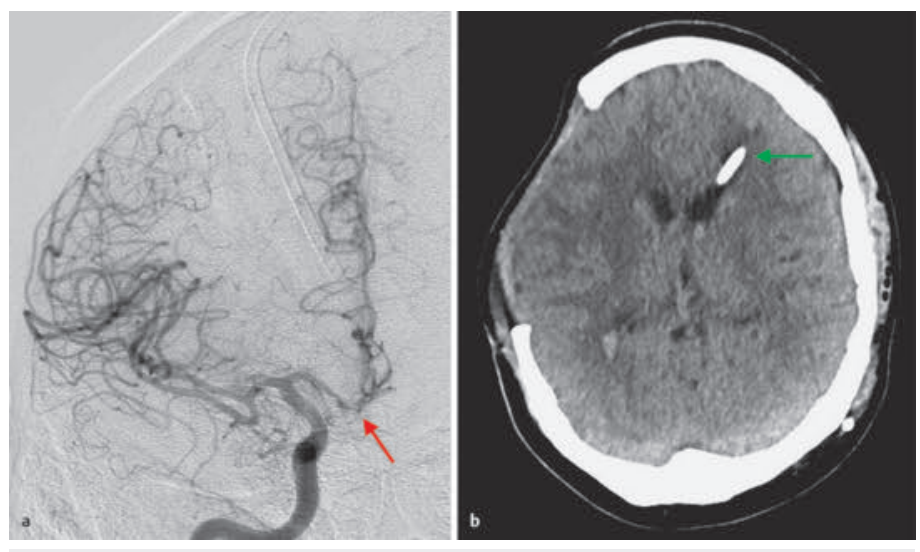

-Abb. 4 Verlauf nach Subarachnoidalblutung und Clipping des Aneurysmas. a Digitale Subtraktionsangiografie: In der postoperativen Kontrolluntersuchung ist das Aneurysma vollständig ausgeschaltet (roter Pfeil). b Die Anlage eines ventrikuloperitonealen Shunts zur dauerhaften Liquorableitung ist erforderlich (grüner Pfeil). durch erhöhten intrakraniellen Druck und Ausschüttung inflammatorischer Proteine (vor allem Interleukin-6) diskutiert. Abgegrenzt von dem rein neurogenen Lungenödem wird das Lungenödem infolge kardialer Dekompensation sowie jenes, das bedingt durch die induzierte Hypertonie als Folge der DCl-Therapie auftritt.

Die Behandlung des neurogenen Lungenödems erfolgt primär durch die Behandlung der SAB und des erhöhten ICP. Die eigentliche Therapie des neurogenen Lungenödems weicht im Wesentlichen nicht von der Therapie eines Lungenödems anderer Ursache ab. Es kommen Diuretika zum Einsatz, nichtinvasive (NIV) Beatmung oder - wenn erforderlich - invasive Beatmung. Einzig bei der Therapie mit vasodilatatorischen Medikamenten, wie Nitroglycerin, welche bevorzugt beim kardialen Lungenödem eingesetzt werden, ist Vorsicht geboten: Diese können mit einer starken Blutdrucksenkung einhergehen und so die Aufrechterhaltung eines ausreichenden zerebralen Perfusionsdrucks erschweren.

\section{FALLBEISPIEL}

\section{Postoperativer Verlauf}

Postoperativ wird der Patient zunächst auf der neurochirurgischen Intensivstation betreut, wo er zeitgerecht extubiert werden kann. Nach der Operation misst man keine Erhöhungen des intrakraniellen Druckes mehr. Der Patient leidet unter starken Kopfschmerzen sowie Übelkeit, welche sich nur langsam bessern, zeigt aber weiterhin kein fokalneurologisches Defizit. Die täglich durchgeführten Doppler-Untersuchungen zeigen keine Erhöhung der Flussgeschwindigkeit in den Hirngefäßen als Hinweis für einen zerebralen Vasospasmus. Nach 10 Tagen kann der Patient auf die Überwachungsstation verlegt werden. In der postoperativ durchgeführten Kontroll-DSA ist das Aneurysma vollständig ausgeschaltet (roter Pfeil in Abb. 4a), Hinweise auf hämodynamisch wirksame Vasospasmen finden sich nicht. Das Entwöhnen von der externen Ventrikeldrainage gelingt nicht, sodass ein ventrikuloperitonealer Shunt zur dauerhaften Liquorableitung angelegt wird (grüner Pfeil in A Abb.4b). Nach Shuntanlage wird der Patient vollständig auf Stationsebene mobilisiert und zur Nachsorge in eine neurologische Frührehabilitation verlegt.

\section{Nachbehandlung}

Die Intensivtherapie sollte auch bei gut beurteilbaren Patienten fortgesetzt werden, bis die Spasmusphase vorbei ist oder der Patient auf einer Intermediate-Care-Einheit hinreichend beurteilt werden kann. 
Merke

Jeder Patient mit einer SAB sollte nach Beendigung der Akuttherapie eine Rehabilitationsmaßnahme in Anspruch nehmen - nicht nur Patienten mit evidenten neurologischen Ausfällen oder protrahierten Verläufen.

Die SAB verursacht bei bis zu zwei Dritteln der Patienten teils schwere mnestische und/ oder kognitive Defizite. Häufige psychische Folgeerscheinungen der SAB sind Depressionen, Phobien oder Zwangsstörungen. Nur ein Drittel der Patienten kehrt an den alten Arbeitsplatz zurück. Die Scheidungsrate ist bei Patienten mit SAB im Vergleich zur Normalbevölkerung deutlich erhöht. Eine frühzeitige neuropsychologische Untersuchung und gerichtete Psychotherapie kann Lebensqualität und Rückkehr in das soziale und Arbeitsleben für die Patienten positiv beeinflussen [21].

\section{KERNAUSSAGEN}

- Die aneurysmatische Subarachnoidalblutung ist ein Notfall, der einer sofortigen Behandlung in einem neurovaskulären Zentrum bedarf.

- Bei Verdacht auf Subarachnoidalblutung sollte als Standarddiagnostik ein natives Schädel-CT durchgeführt werden.

- Bei einem Aneurysmanachweis als Blutungsquelle sollte eine Versorgung innerhalb der ersten 48 Stunden angestrebt werden.

- Der beste Weg der Aneurysmaversorgung sollte interdisziplinär zwischen einem interventionell tätigen Neuroradiologen und einem Neurochirurgen für jeden Fall individuell festgelegt werden.

- Die häufigste Komplikation der SAB ist der Hydrozephalus.

- Innerhalb der ersten 14 Tage nach Blutung besteht die Gefahr eines verzögerten neurologischen Defizits, sodass in dieser Zeit eine engmaschige Überwachung erforderlich ist.

- In bis zu zwei Dritteln der Fälle leiden die Patienten auch nach der Akutphase unter schweren mnestischen und kognitiven Defiziten.

\section{Interessenkonflikt}

Herr PD Dr. Oliver Müller war PI für Egde Therapeutics, Frau Dr. Müller hat keinen Interessenkonflikt.

\section{Autorinnen / Autoren}

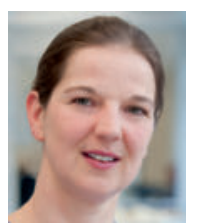

\section{Daniela Müller}

Dr. med., 1993-2000 Studium der Humanmedizin an der Charité Berlin und der Julius-Maximilians-Universität Würzburg. 2000-2007 Facharztausbildung Neurochirurgie, Zusatzbezeichnung Intensivmedizin 2010. Seit 2010
Leiterin der Neurochirurgischen Intensivstation am Universitätsklinikum Essen. Schwerpunkte: Behandlung der SAB und des Schädel-HirnTraumas, psychologische Folgen der Intensivtherapie bei Patienten.

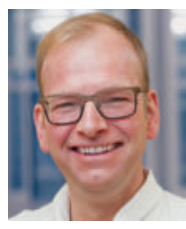

\section{Oliver Müller}

Professor Dr. med., 1993-1999 Studium der Humanmedizin an der Universität Gesamthochschule Essen. 1999-2006 Facharztausbildung Neurochirurgie, Zusatzbezeichnung Intensiv- und Notfallmedizin 2008. Seit März 2013 Stellvertretender Klinikdirektor der Neurochirurgie am Universitätsklinikum Essen. Schwerpunkte: vaskuläre Neurochirurgie, endoskopische Schädelbasis-Chirurgie, pädiatrische Neurochirurgie.

\section{Korrespondenzadresse}

\author{
Dr. med. Daniela Müller \\ Klinik für Neurochirurgie \\ Universitätsklinikum Essen \\ Hufelandstraße 55 \\ 45147 Essen \\ E-Mail: d.mueller@uk-essen.de
}

Wissenschaftlich verantwortlich gemäß

Zertifizierungsbestimmungen

Wissenschaftlich verantwortlich gemäß

Zertifizierungsbestimmungen für diesen Beitrag ist Dr. med.

Daniela Müller, Essen.

\section{Erstveröffentlichung}

Dieser Beitrag wurde erstveröffentlicht in: Anästhesiol Intensivmed Notfallmed Schmerzther 2018; 53: 654-667.

Literatur

[1] Macdonald RL, Schweizer TA. Spontaneous subarachnoid haemorrhage. Lancet 2017; 389: 655-666. doi: 10.1016/S01406736(16)30668-7

[2] Connolly ES jr., Rabinstein AA, Carhuapoma JR et al. Guidelines for the management of aneurysmal subarachnoid hemorrhage: a guideline for healthcare professionals from the American Heart Association/American Stroke Association. Stroke 2012; 43: 1711-1737. doi: 10.1161/STR.0b013e3182587839

[3] Passier PE, Visser-Meily JM, Rinkel G] et al. Life satisfaction and return to work after aneurysmal subarachnoid hemorrhage. J Stroke Cerebrovasc Dis 2011; 20: 324-329. doi:10.1016/j. jstrokecerebrovasdis.2010.02.001

[4] Mills JN, Mehta V, Russin J et al. Advanced imaging modalities in the detection of cerebral vasospasm. Neurol Res Int 2013; 2013: 415960. doi:10.1155/2013/415960

[5] Mueller OM, Schlamann M, Mueller D et al. Intracranial aneurysms: optimized diagnostic tools call for thorough interdisciplinary treatment strategies. Ther Adv Neurol Disord 2011; 4: 267-279. doi:10.1177/1756285611415309 
[6] Ringelstein A, Mueller O, Monninghoff $\mathrm{C}$ et al. 3D rotational angiography after non-traumatic SAH. Rofo 2014; 186: 675679. doi:10.1055/s-0033-1356325

[7] Ringelstein A, Mueller O, Goericke SL et al. Benefit of second catheter angiography in patients with nontraumatic subarachnoidal hemorrhage. Clin Neuroradiol 2015; 25: 13-17. doi:10.1007/s00062-013-0271-5

[8] Ahmadian A, Mizzi A, Banasiak M et al. Cardiac manifestations of subarachnoid hemorrhage. Heart Lung Vessel 2013; 5: 168-178

[9] Garfinkle AM, Danys IR, Nicolle DA et al. Terson's syndrome: a reversible cause of blindness following subarachnoid hemorrhage. J Neurosurg 1992; 76: 766-771. doi:10.3171/ jns.1992.76.5.0766

[10] Ohkuma H, Tsurutani H, Suzuki S. Incidence and significance of early aneurysmal rebleeding before neurosurgical or neurological management. Stroke 2001; 32: 1176-1180

[11] Whitfield PC, Kirkpatrick PJ. Timing of surgery for aneurysmal subarachnoid haemorrhage. Cochrane Database Syst Rev 2001; (2): CD001697. doi:10.1002/14651858.CD001697

[12] Suarez JI, Zaidat OO, Suri MF et al. Length of stay and mortality in neurocritically ill patients: impact of a specialized neurocritical care team. Crit Care Med 2004; 32: 2311-2317

[13] Treggiari MM; Participants in the International Multi-disciplinary Consensus Conference on the Critical Care Management of Subarachnoid Hemorrhage. Hemodynamic management of subarachnoid hemorrhage. Neurocrit Care 2011; 15: 329335. doi:10.1007/s12028-011-9589-5

[14] Rowland M], Hadjipavlou G, Kelly M et al. Delayed cerebral ischaemia after subarachnoid haemorrhage: looking beyond vasospasm. Br J Anaesth 2012; 109: 315-329. doi:10.1093/ bja/aes264

[15] Vergouwen MD, Vermeulen M, van Gijn J et al. Definition of delayed cerebral ischemia after aneurysmal subarachnoid hemorrhage as an outcome event in clinical trials and observational studies: proposal of a multidisciplinary research group. Stroke 2010; 41: 2391-2395. doi:10.1161/STROKEAHA. 110.589275

[16] Simard JM, Aldrich EF, Schreibman D et al. Low-dose intravenous heparin infusion in patients with aneurysmal subarachnoid hemorrhage: a preliminary assessment. J Neurosurg 2013; 119: 1611-1619. doi:10.3171/2013.8.JNS1337

[17] Chen S, Luo J, Reis C et al. Hydrocephalus after subarachnoid hemorrhage: pathophysiology, diagnosis, and treatment. Biomed Res Int 2017; 2017: 8584753. doi:10.1155/2017/ 8584753

[18] Bardutzky J, Witsch J, Juttler E et al. EARLYDRAIN - outcome after early lumbar CSF-drainage in aneurysmal subarachnoid hemorrhage: study protocol for a randomized controlled trial. Trials 2011; 12: 203. doi:10.1186/1745-6215-12-203

[19] Jabbarli R, Bohrer AM, Pierscianek D et al. The CHESS score: a simple tool for early prediction of shunt dependency after aneurysmal subarachnoid hemorrhage. Eur J Neurol 2016; 23: 912-918. doi:10.1111/ene.12962

[20] Muroi C, Keller M, Pangalu A et al. Neurogenic pulmonary edema in patients with subarachnoid hemorrhage. J Neurosurg Anesthesiol 2008; 20: 188-192. doi:10.1097/ANA.0b01 3 e3181778156

[21] Rinkel G], Algra A. Long-term outcomes of patients with aneurysmal subarachnoid haemorrhage. Lancet Neurol 2011; 10: 349-356. doi:10.1016/S1474-4422(11)70017-5

\section{Bibliografie}

DOI https://doi.org/10.1055/a-0835-9617 Fortschr Neurol Psychiatr 2019; 87: 192-204 (c) Georg Thieme Verlag KG Stuttgart · New York ISSN 0720-4299 


\section{Punkte sammeln auf GME.thieme.de}

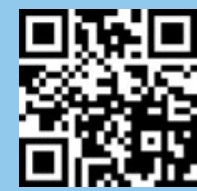

Diese Fortbildungseinheit ist in der Regel 12 Monate online für die Teilnahme verfügbar.

Den genauen Einsendeschluss finden Sie unter https://eref.thieme.de/CXCIQ]7.

Sollten Sie Fragen zu Online-Teilnahme haben, finden Sie unter https://cme.thieme.de/hilfe eine ausführliche Anleitung. Wir wünschen viel Erfolg beim Beantworten der Fragen!

Unter https://eref.thieme.de/CXCIQJ7 oder über den QR-Code kommen Sie direkt zur Startseite des Wissenstests.

VNR 2760512019156641993

\section{Frage 1}

Die Diagnostik bei einem Patienten im klinischen Zustand WFNS I/II nach Aufnahme im Krankenhaus bei Verdacht auf und/oder Nachweis einer akuten SAB sollte beinhalten....

A Natives CCT

B Gegebenenfalls Lumbalpunktion

C CTA oder DSA

D Neurologische Untersuchung

E Invasives Monitoring

\section{Frage 2}

Eine Subarachnoidalblutung ist:

A eine Blutung in das Hirnparenchym

B meist durch eine venöse Problematik verursacht

C eine häufige Ursache eines sogenannten Schlaganfalls

D bedarf zunächst keiner weiteren Abklärung

E eine lebensbedrohliche Erkrankung und bedarf der sofortigen intensivmediznischen Überwachung und Therapie

\section{Frage 3}

Was gehört nicht zur typischen Symptomatik bei SAB?
A Vernichtungskopfschmerz
B Übelkeit und Erbrechen
C Bewusstseinstrübung
D Querschnittssymptomatik
E Krampfanfälle

\section{Frage 4}

Ein Aneurysma der intrakraniellen Arterien als Ursache einer Subarachnoidalblutung

A Wird im nativen CCT diagnostiziert und beurteilt

B Kann sich vor dem Indexereignis durch eine Warnblutung bemerkbar machen

C Tritt in 2/3 der Fälle multiple auf

D Verursacht stets begleitende Hirnnervenausfälle

E Ist eine Anlage-bedingte Entwicklungsstörung

\section{Frage 5}

Die Versorgung des Aneurysmas

A Hat keinen Vorrang und kann im Verlauf erfolgen

B Erfolgt immer endovaskulär

C sollte in einem Zentrum mit neurovaskulärer Expertise nach interdisziplinärer Besprechung erfolgen

D Muss im erstbehandelnden Krankenhaus erfolgen

E Verhindert Komplikationen wie Hydrocephalus oder Vasospasmus!

\section{Frage 6}

Was gehört nicht zu den typischen Komplikationen der SAB?

A Herzrhythmusstörungen

B Harnwegsinfekt

C Hydrozephalus

D Hyponatriämie

E Terson-Syndrom

\section{Frage 7}

Welche Aussage ist falsch? Kardiale Komplikationen im Rahmen der SAB ...

A sind auf exzessive Katecholaminausschüttung im Rahmen der SAB zurückzuführen.

B können bis hin zur Herzinsuffizienz führen.

C sind häufige Begleitkomplikationen.

D bedürfen keiner Therapie.

E sind mit einem schlechteren Outcome vergesellschaftet.

\section{Frage 8}

Die delayed cerebral ischaemia
A Ist ein Synonym für Vasospasmus
B Tritt meist direkt postoperativ auf
C Wird mittels Dopplersonographie der Hirngefäße diagnostiziert
D Ist eine klinische Diagnose
E Wird mit Antiepileptika behandelt 


\section{Punkte sammeln auf CME.thieme.de}

Fortsetzung $\ldots$

\section{Frage 9}

Welche Aussage ist richtig? Bei der intensivmedizinischen SAB-Therapie ...
A sollte auf konsequente Senkung von Fieber geachtet werden.
B ist es wichtig, den Blutdruck nicht anzuheben.
C sollte die Hyponatriämie mit Flüssigkeitsrestriktion behan- delt werden.
D ist eine Thromboseprophylaxe nicht notwendig.
E dürfen keine Katecholamine verwendet werden.

\section{Frage 10}

Welche Aussage ist richtig? Nach einer SAB ...
A haben viele der Patienten ein persistierendes kognitives Defizit.
B kehren die meisten Patienten an ihren alten Arbeitsplatz zurück.
C sind Rehabilitationsmaßnahmen meist nicht notwendig.
D sind neurologische Defizite nicht zu erwarten.
E sind üblicherweise ambulante Rehamaßnahmen ausreichend. 